\title{
Supply-side and trickle-down therapy
}

Children's Hospital Research Foundation, Division of Human Genetics, Cincinnati, Ohio 45229-3039, USA

Address correspondence to: Gregory A. Grabowski, Children's Hospital Research Foundation, Division of Human Genetics, 3333 Burnet Avenue, Cincinnati, Ohio 45229-3039, USA. Phone: (513) 636-7290; Fax: (513) 636-2261; E-mail: grabg0@chmcc.org

The glycosphingolipid (GSL) storage diseases have occupied a position of importance in biochemistry, intermediary metabolism, genetics, and gene product therapy disproportionate to their frequency in the population. In this issue of the JCI, Liu et al. (1) extend this history by providing "proof-of-principle" for potential new therapies of lysosomal storage diseases (LSDs) and raise general issues relevant to phenotypic expression. In essence, Liu et al. show that "nonessential" metabolic intermediates - selected GSLs with singular major synthetic and degradative pathways - can be eliminated and thereby decrease storage without apparent adverse effects. Although genetically based, Liu et al.'s approach is conceptually identical to the successful dietary restriction therapies for amino/organic acid disorders and to the inhibitor therapies for dyslipidemias.

With a few notable exceptions, GSL storage diseases result from single

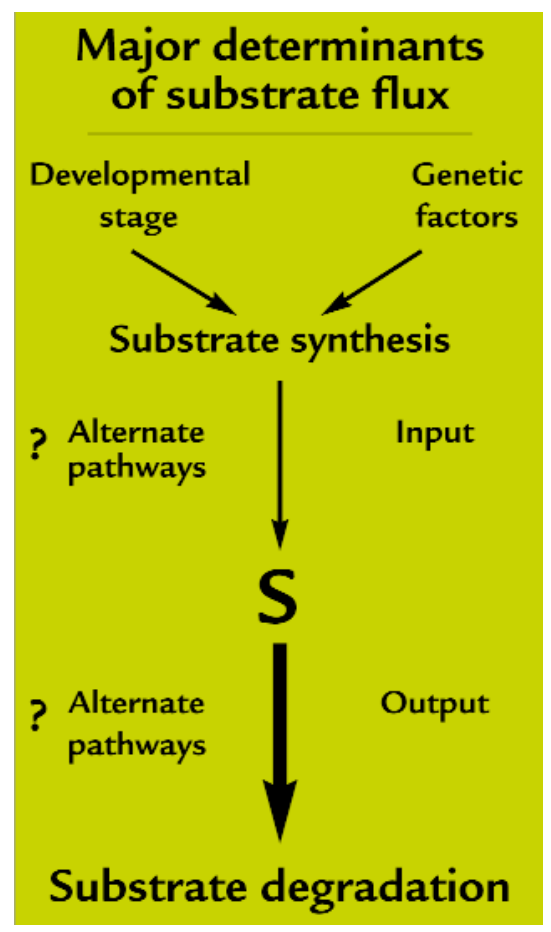

gene-single enzyme defects, and the consequent accumulation of hallmark substrates for specific phenotypes. Radin (2) suggested that the phenotypes developed from substrate flux disruptions that in turn depend on the inflow and outflow rates of intermediates, and directed attention to the supply side (Fig. 1). If either rate is altered beyond a threshold level, intermediates will accumulate and produce phenotypes. This threshold model (3) implies that the flux through the metabolic pathway can be altered by increasing the amount of residual enzyme (increase the trickle) or by decreasing the substrate input (drop the supply). This model encompasses the regional, organ, and cell levels, because of specific differential metabolism. Also, the degree of tissue involvement is affected by the specific substrate flux rates in various cellular types. A good correlation exists between the level of in situ residual enzymatic activity in cell cultures, and the phenotypic severity of LSDs (4). Simplistically, the less residual enzyme activity, the more rapid the accumulation of substrate and the more severe the phenotype. Augmentation of the deficient enzyme to levels above threshold should correct substrate flux, and, if reversible, the phenotype. The approaches of genebased and enzyme therapies have focused nearly exclusively on the outflow side.

The $\beta$-hexosaminidases are essential for degradation of the GSLs, $\mathrm{G}_{\mathrm{M} 2}$ ganglioside and globoside. Mutations in the $\alpha$-chain lead to $\beta$-hexosaminidase A $\left(\alpha_{2} \beta\right)$ deficiencies and the Tay Sachs variants with $\mathrm{CNS}_{\mathrm{M} 2}$ ganglioside storage. Mutations of $\beta$-chains produce deficien- cies of $\beta$-hexosaminidases $A$ and $B\left(\beta_{3}\right)$ and the "Sandhoff phenotypes" with $\mathrm{CNS} \mathrm{G}_{\mathrm{M} 2}$ ganglioside and visceral globoside storage (5). The CNS phenotypes result from the cellular effects, albeit poorly characterized, of the accumulated $\mathrm{G}_{\mathrm{M} 2}$ ganglioside. Sandhoff mice have progressive motor behavior loss and shortened life span (6). Liu et al. substantially, but not completely, obviated this Sandhoff phenotype by breeding in an amorph for the enzyme $\beta 1-4-N$-acetylgalactosaminyltransferase (GalNAcT) that is essential for $\mathrm{G}_{\mathrm{M} 2}$ ganglioside and globoside synthesis. Except for male sterility and late CNS storage of oligosaccharides, the double homozygous mutants (Hexa/b $b^{-/-}$GalNAcT $\left.T^{-1}\right)$ attained a nearly normal phenotype at the functional, histological, and biochemical levels.

These remarkable results offer a classic example of genetic epistasis, i.e., a secondary genetic change completely obscuring the genetic propensity to develop the primary phenotype. Surprisingly, the GalNAcT $T^{-/}$mice lack complex GSLs throughout all of development and are nearly normal. Given the apparent role of complex GSLs as membrane components in cellular interaction and recognition as well as other major biological phenomenon (5), the implication that these lipids are just decorative gives one pause. Perhaps the behavioral or biochemical tests are too insensitive or are inappropriate to detect abnormalities. Nevertheless, gross dysfunction might have been anticipated. Such a free lunch seems doubtful. Are there alternative, compensatory pathways? The alternative explanation that mice are mice and men are men is not

\section{Figure 1}

Substrate flux is determined by various input and output rates including substrate synthesis, but other factors, including alternative metabolic pathways, environmental exposures, cell type, or tissue region, etc., can significantly influence this rate. " $\mathrm{S}$ " indicates a steady state level of substrate that is not necessarily normal. A significant issue is the level of substrate that is compatible with normalcy. The rate of " $\mathrm{S}$ " increase determines the age of onset and progression of metabolic diseases. 
without merit, but it must be viewed in the context of the great genetic and complex GSL similarities between these two species. The preservation of complex synthetic machinery for such apparently "non-essential" metabolites would have profound evolutionary and biologic implications.

In human $\mathrm{G}_{\mathrm{M} 2}$ gangliosidoses, storage is readily observed by 6 weeks of gestation. Thus, postnatal therapy for GSLs could be seriously limited by preexisting irreversible disease. Liu et al.'s Hex $a / b^{-/-}$GalNAcT $T^{4-}$ mice had significant clinical and biochemical abnormalities. This suggests the need for continuous, complete inhibition of the synthetic pathway that may not be achievable by drug therapy, and complete prevention of progressive CNS disease may not be possible in postnatal humans. So, the postnatal approach may lead to late onset variants. Institution of prenatal substrate deprivation therapy involves two patients, the fetus and the mother. Therapy would be initiated shortly after conception, and maternal exposure would exist throughout the pregnancy. The prenatal strategy presents the ethical conundrum of potential harm to the mother for benefit to the fetus.

The greatest therapeutic effects of substrate deprivation for GSLs as well as more general applications could be in the hypomorphs, i.e., affected patients with significant residual enzyme and a late (milder) phenotype. Decreasing substrate inflow might allow for less aberrant flux, further delaying the phenotype, and permitting longer-term functionality. Addition of enzyme replacement and/or genebased therapies could provide additional corrections. The latter approaches are formidable challenges for CNS diseases, because delivery of functional macromolecules to the brain has proved difficult. Such effective therapies may provide hope to the families affected by substrate overload diseases.

The basic implications and opportunities also are great. Development of a cadre of animals with specific defects in GSL or other synthetic pathways would provide critical reagents for dissecting and understanding metabolic pathophysiology and would be important additions to their functional genomics. Although amorphs provide "proof of principal," hypomorphs may provide greater biological insight. The use of conditional expression systems for willful modulation of the relative expression of various genes in vivo (7) provide extraordinarily powerful tools to examine the biology of GSL and many other metabolic intermediates. More generally, a fundamental issue in genetics is the interaction of hypomorphic/allelic variants at several loci whose combined effects lead to differential phenotypes as a result of genetic epistasis or other poorly defined gene-gene interactions. Conditional expression systems allowing independent titration of gene expression would provide exquisite tools for critical analysis of gene-gene interaction at an organismal level. These broader applications to a variety of pathways provide potential links between statistical and functional genomics and are the fundamental implications of Liu et al.'s work.

1. Liu, Y., et al. 1999. A genetic model of substrate deprivation therapy for a glycosphingolipid storage disorder. J. Clin. Invest. 103:497-505.

2. Radin, N.S. 1982. Inhibitors and stimulators of glucocerebroside metabolism. Prog. Clin. Biol. Res. 95:357-383.

3. Conzelmann, E., and Sandhoff, K. 1983. Partial enzyme deficiencies: residual activities and the development of neurological disorders. Dev. Neurosci. 6:58-71.

4. Leinekugel, P., Michel, S., Conzelmann, E., and Sandhoff, K. 1992. Quantitative correlation between the residual activity of beta-hexosaminidase A and arylsulfatase A and the severity of the resulting lysosomal storage disease. Hum. Genet. 88:513-523.

5. Gravel, R.A., et al. 1995. The GM2 gangliosidoses. In The metabolic and molecular bases of inherited disease. C.R. Scriver, A.L. Beaudet, W.S. Sly, and D. Valle, editors. McGraw-Hill. New York, NY. 2839-2882.

6. Sango, K., et al. 1995. Mouse models of Tay-Sachs and Sandhoff diseases differ in neurologic phenotype and ganglioside metabolism. Nat. Genet. 11:170-176.

7. Gossen, M. and Bujard, H. 1992. Tight control of gene expression in mammalian cells by tetracycline-responsive promoters. Proc. Natl. Acad. Sci. U.S.A. 89:5547-5551. 